\section{A long-term increase in the fluid intelligence of English children}

\author{
Richard Lynn, Susan L. Hampson \\ \& Judith C. Mullineux \\ University of Ulster, Coleraine, County Londonderry BT52 1SA, UK
}

In the early decades of the century a number of geneticists and psychologists believed that intelligence in the economically advanced nations was in secular decline $e^{1,2}$. Contrary to this expectation, recent data for a number of countries have shown that intelligence has been increasing at rates far greater than hitherto considered probable. A compilation of this data by Flynn for 14 economically advanced nations has shown that intelligence quotient (IQ) rises have generally been within the range of 2-12 IQ points per decade ${ }^{3}$. These recent studies raise several questions, among which are precisely what abilities have been increasing over time; and whether existing data are correct in suggesting that the secular rise in Britain is lower than that in other countries. We report here that in English children there has been an increase over the past 50 years of 12.42 IQ points, averaging 2.48 points per decade. The increase has been in fluid intelligence, the mental power that underlies the acquisition of cognitive skills, rather than in crystallized intelligence, which represents the cognitive skills acquired in a particular culture.

To address the question of which cognitive skills have been increasing over time, we adopt the classical British hierarchical model proposed by Spearman and developed by Burt and Cattell. This postulates a broad factor of general intelligence (Spearman's $g$ ), possibly identifiable with the efficiency of neural processing; two broad factors of the verbal and visuospatial abilities: and some 25-30 narrow primary abilities. Cattell's elaboration of the model splits Spearman's $g$ into two general factors identified as fluid intelligence (mental power) and crystallized intelligence (developed cognitive skills) ${ }^{4}$. Fluid intelligence is measured by the culture fair intelligence tests, constructed by Cattell to minimize cultural and educational content and consisting of problems presented in picture and diagram format. The first of these tests was the Cattell Non-Verbal and was standardized for English school children in 1935 (ref. 5). In 1936 the test was administered to all 9-11-year-old children in the city of Leicester. The mean IQ of this sample was 100.49 (ref.6), which agrees closely with the mean of the 1935 standardization sample and indicates a well standardized test for the measurement of fluid intelligence of English children of the mid-1930s.

In 1985, half a century after the original standardization, we administered the same test to a sample of 1,029 English 9-11year-old children, drawn from 14 primary schools selected on the advice of headteachers as socially representative and situated in Leeds, Newcastle, Birmingham, Suffolk, Northamptonshire, Cambridgeshire and Sussex. The testing was carried out in the early summer and there were virtually no absentees. Children in special schools for the educationally subnormal were not included in the 1935 sample and were therefore also excluded from the 1985 sample. In 1985 only $1.3 \%$ of children attended such schools and the effect of this group on the mean IQ of the total population is negligible?

The results of the 1985 survey showed that in all the schools the children obtained substantially higher IQ scores than the 1935 standardization sample. Working on a standard deviation of 15 , the 1985 cohort obtained a mean IQ of 112.42 , representing an increase of 2.48 IQ points per decade over the 50 year period. The difference between the means of the 1935 and 1985 samples is statistically highly significant (in Student's $t$-test, $t=18.2$; $P<0.001$ ). There has therefore been an appreciable rise in the IQ of English children in the past 50 years that can be attributed to an increase in fluid intelligence.
In his 14-nation review of secular increases in intelligence, Flynn suggests that the increase in Britain seems to be smaller than that in other economically advanced nations ${ }^{3}$. This suggestion is based on three sets of data, namely Raven's Progressive Matrices (1938-1979), Cattell's Non-Verbal Test (1936-1949) and the Moray House Test (1932-1947). All three studies produced increases of less than 2 IQ points per decade, whereas virtually all results for other economically advanced nations have shown increases of the order of 2-12 IQ points per decade.

All three of these studies span the years of the Second World War. It may be that this had a depressing effect on intelligence and therefore on the rate of gain. This possibility can be tested, because Cattell administered his test to all 10-year-olds in the city of Leicester in 1949 (ref. 6). If the 1949 mean IQ is used as a base, the increase from 1949 to 1985 was 3.16 IQ points per decade. This figure is appreciably greater than the rise of $\sim 0.75$ IQ points per decade for the period 1935-1949. We therefore conclude that the low rates of gain in Britain noted by Flynn are attributable to the inclusion of the Second World War years in the existing studies and that in the post-war period the rate of gain of $3.16 \mathrm{IQ}$ points per decade is within the normal range for other economically advanced nations.

Our results clearly indicate that there has been a substantial increase in Cattell's fluid intelligence in English children over the last half-century. This suggests that the rises in the mean scores on the various tests which have been found in different studies over a wide range of countries are not primarily due to increases in learned cognitive skills resulting from improvements in education and the cultural environment, as proposed by Teasdale and Owen ${ }^{8}$, but in more basic and general mental ability. For further progress in this field it would be useful to investigate and quantify the secular increases that have taken place in other components of the Spearman-Burt-Cattell model.

Received 6 May; accepted 15 July 1987.

1. Fisher, R. A. The Genetical Theory of Natural Selection (Dover, London, 1929).

2. Burt, C. L. Intelligence and Fertility (Hamilton, London, 1946).

3. Flynn, J. R. Psychol, Bull, 101, 171-191 (1987)

4. Cattell, R. B. Abilities: Their Structure, Growth and Action (Houghton Mifflin, Boston, 1971).

5. Cattell, R. B. A Guide to Mental Testing (London University Press, 1948).

6. Cattell, R. B. Eugen. Rev. 42, 136-148 (1950).

Statistical Bulletin (Dept. of Education and Science, London, 1987).

8. Teasdale, T. W. \& Owen, D. R. Nature 325, 119-121 (1987).

\section{Adaptive suicidal behaviour in pea aphids}

\section{Murdoch K. McAllister \& Bernard D. Roitberg}

Behavioural Ecology Research Group and Centre for Pest Management, Department of Biological Sciences, Simon Fraser University, Burnaby, British Columbia V5A 1S6, Canada

The host suicide hypothesis ${ }^{1}$, which derives from inclusive fitness theory ${ }^{2}$, postulates that parasitized individuals in spatially aggregated populations consisting of close kin may actively enhance their probability of dying. The fitness cost associated with suicide becomes negligible when infection by a parasitoid causes the expected reproduction of the host to approach zero. But the host will benefit from suicide, if by its death (and that of its parasite) the level of subsequent parasitism in its kin is reduced relative to that in non-kin. Although conceptually appealing, host suicide has not yet been clearly demonstrated ${ }^{3}$. Here we report that pea aphid (Acyrthosiphon pisum) parasitized by the braconid wasp Aphidius ervi, exhibit apparent suicidal behaviour in response to both aphid alarm pheromone and approaching coccinellid (ladybird beetle) predators. We believe this to be the first convincing evidence in support of the host suicide hypothesis. 